EESTI NSV TEADUSTE AKADEEMIA TOIMETISED.

FUUSIKA * MATEMAATIKA

ИЗВЕСТИЯ АКАДЕМИИ НАУК ЭСТОНСКОИ ССР. ФИЗИКА * МАТЕМАТИКА

PROCEEDINGS OF THE ACADEMY OF SCIENCES OF THE ESTONIAN SSR.

PHYSICS * MATHEMATICS

$1984,33,4$

B. BAATMAHH

удК 519.2 !

\title{
О МИНИМАКСНОМ ОЦЕНИВАНИИ СОСТОЯНИЙ ЛИНЕИНЫХ СТОХАСТИЧЕСКИХ СИСТЕМ
}

\author{
(Представил Н. Алумяэ)
}

Рассматривается минимаксное оценивание состояний линейной стохастической системы рекуррентными уравнениями типа Калмана-Бюси в случае, когда информация о переходных матрицах системы ограничивается заданием определенных условий. Сначала рассматривается система с дискретным временем, затем с непрерывным.

В литературе $\left[{ }^{1-3}\right]$ в основном исследовано минимаксное оценивание состояний стохастической системы для случаев, когда вероятностные характеристики возмущений и начального состояния системы точно не известны.

1. Пусть стохастическая система с дискретным временем описывается уравнением состояний

$$
x(t+1)=A(t) x(t)+e(t)
$$

и уравнением наблюдений

$$
y(t)=C(t) x(t)+v(t), \quad t \in T,
$$

где $T=\{0,1,2, \ldots\}, x-n$-мерный вектор состояний системы, $y-$ $m$-мерный вектор выходных переменных и $e, v-$ последовательности некоррелированных случайных векторов с нулевыми математическими ожиданиями и ковариациями

$$
\mathbf{E}\left(e(s) e^{\mathrm{T}}(t)\right)=R_{1}(t) \delta(s-t), \quad \mathbf{E}\left(v(s) v^{\mathrm{T}}(t)\right)=R_{2}(t) \delta(s-t) .
$$

Через Е мы обозначили оператор математического ожидания, через $\delta$ - функцию Кронекера; верхний индекс Т означает транспонирование. Предположим, что $R_{2}(t)$ - положительно определенная матрича, начальное состояние $x(0)$ системы (1) не коррелировано с $e$ и $v$, имеет математическое ожидание $x_{0}$ и ковариационную матрицу $R_{0}$. Допустим, что матрицы $C(t), t \in T$, известны, а информация о переходных матрицах ограничивается заданием условий $A(s) \in H_{s}, s \in T$, где $H_{s}$ определено через заданные $n \times n$-матрицы $A_{0}(s)$ как

$$
H_{s}=\left\{A: z^{\mathrm{T}}\left(A^{\mathrm{T}} A-A_{0}^{\mathrm{T}}(s) A_{0}(s)\right) z \leqslant 0, \quad \mathrm{~V} z \in R^{n}\right\}, \quad s \in T .
$$

Пусть процесс $x(t), t=0,1,2, \ldots$, недоступен наблюдению, наблюдать можно лишь значения $y(s), s=0,1, \ldots, t$, несущие в себе неполную информацию о значениях $x(t+1)$. Требуется в каждый момент $t+1$ оценить значения $x(t+1)$ по реализации $y(s), s=0,1, \ldots, t$.

Если последовательность $A(s), s \in T$, точно задана, то линейная оптимальная в среднеквадратическом смысле оценка вектора $x(t+1)$ определяется рекуррентным уравнением Қалмана-Бюси 


$$
\hat{x}(t+1)=A(t) \hat{x}(t)+K(t)(y(t)-C(t) \hat{x}(t)), \quad \hat{x}(0)=x_{0},
$$

где $K(s), s=\overline{0, t}$, рекуррентно определенные $n \times m$-матрицы.

Рассмотрим минимаксные оценки в случае, когда в системе (1) переходные матрицы $A(s), s \in T$, точно не известны. Обозначим через $F_{t+1}\left(H_{0}, H_{1}, \ldots, H_{t}\right)$ класс оценок вектора $x(t+1)$, определяемых уравнением (4), где $A(s) \in H_{s}, K(s) \in R^{n \times m}, s=\overline{0, t}$.

Оценку $\hat{x_{0}}(t+1)$ вектора $x(t+1)$ из класса оценок $F_{t+1}\left(H_{0}, H_{1}, \ldots\right.$ $\left.\ldots, H_{t}\right)$ будем называть минимаксной, если

$$
\mathbf{E}\left\|\hat{x}_{0}(t+1)-x(t+1)\right\|^{2}=\min _{K(s) \in R} \max _{n \times m} \mathbf{E}\|\hat{x}(t+1)-x(t+1)\|^{2} .
$$

Определенная так минимаксная оценка в отсутствие неопределенности по матрицам $A(s), s=\overline{0, t}$, совпадает с оценкой Калмана-Бюси.

Те орем а 1. Минимаксная оценка состояний системы (1) в момент времени $t+1$ по наблюдениям $y_{0}, y_{1}, \ldots, y_{t}$ удовлетворяет рекуррентному уравнению

$$
\begin{aligned}
\hat{x}(t+1) & =A_{0}(t) \hat{x}(t)+K(t)(y(t)-C(t) \hat{x}(t)) ; \\
\hat{x}(0) & =x_{0},
\end{aligned}
$$

где $K(t)=A_{0}(t) P(t) C^{\mathrm{T}}(t)\left(C(t) P(t) C^{\mathrm{T}}(t)+R_{2}(t)\right)^{-1} \quad u P(t)$ определяется уравнением

$$
\begin{gathered}
P(t+1)=A_{0}(t)\left[P(t)-P(t) C^{\mathrm{T}}(t)\left(C(t) P(t) C^{\mathrm{T}}(t)+\right.\right. \\
\left.\left.+R_{2}(t)\right)^{-1} C(t) P(t)\right] A_{0}^{\mathrm{T}}(t)+R_{1}(t), \\
P(0)=R_{0}
\end{gathered}
$$

при этом

$$
\operatorname{tr} P(t)=\min _{K(s) \in R} \max _{n \times m} \operatorname{man}_{s=0, t} \mathbf{E}\|\hat{x}(t) \rightarrow x(t)\|^{2}
$$

Доказ а тельст во. Пусть $A, B$ и $P-n \times n$-матрицы. В дальнейшем мы используем следующие известные свойства матриц: 1) если $c_{1}, c_{2}$ скаляры, то $\operatorname{tr}\left(c_{1} A+c_{2} B\right)=c_{1} \operatorname{tr} A+c_{2} \operatorname{tr} B$; 2) $\operatorname{tr}(A B)=\operatorname{tr}(B A)$; 3) $\operatorname{tr}\left(A P A^{\mathrm{T}}\right)=\sum_{i=1}^{n} a_{i}^{\mathrm{T}} P a_{i}$,где $a_{i}, i=\overline{1, n}$, вектор-строки матрицы $A$;

4) собственные числа матриц $A B$ и $B A$ совпадают; 5) если $A, B$ - симметрические матрицы, причем $B$ неотрицательно определена, то собственные значения матриц $A+B$ и $A$ удовлетворяют неравенству $\lambda_{k}(A+B) \geqslant \lambda_{k}(A), k=\overline{1, n}$.

Из $\left[{ }^{4}\right]$ следует, что выражение оценки $\hat{x}(t+1)$ из класса $F_{t+1}\left(H_{0}\right.$ $\left.H_{1}, \ldots, H_{t}\right)$ может быть записано в виде $\hat{x}(t+1)=A(t)[\hat{x}(t)+$ $+K(t)(y(t)-C(t) \hat{x}(t))], \hat{x}(0)=x_{0}$, где $A(s) \in H_{s}, K(s)-$ произвольная матрица порядка $n \times m, s=\overline{0, t}$.

Преобразуем выражение среднеквадратической ошибки оценки, ис- 
пользуя свойство 2) следа матрицы и учитывая (1), (2). Буквой $I$ обозначим единичную $n \times n$-матрицу.

$$
\begin{aligned}
& \mathbf{E}\|\hat{x}(t+1)-x(t+1)\|^{2}=\mathbf{E}\left[(\hat{x}(t+1)-x(t+1))^{\mathrm{T}}(\hat{x}(t+1)-x(t+1))\right]= \\
= & \operatorname{tr} \mathbf{E}\left[(\hat{x}(t+1)-x(t+1))(\hat{x}(t+1)-x(t+1))^{\mathrm{T}}\right]= \\
= & \operatorname{tr} \mathbf{E}\{[A(t)((I-K(t) C(t))(\hat{x}(t)-x(t))+K(t) v(t))-e(t)] \times \\
\times & {\left.[A(t)((I-K(t) C(t))(\hat{x}(t)-x(t))+K(t) v(t))-e(t)]^{\mathrm{T}}\right\}=} \\
= & \operatorname{tr}\left\{A ( t ) \left[(I-K(t) C(t)) P(t)(I-K(t) C(t))^{\mathrm{T}}+\right.\right. \\
+ & \left.\left.K(t) R_{2}(t) K^{\mathrm{T}}(t)\right] A^{\mathrm{T}}(t)+R_{1}(t)\right\},
\end{aligned}
$$

где $P(t)$ ковариационная матрица ошибки $\hat{x}(t)-x(t)$. Для упрощения записи введем матрицу $S(t)$, определяемую следующим образом:

$$
S(t)=(I-K(t) C(t)) P(t)(I-K(t) C(t))^{\mathrm{T}}+K(t) R_{2}(t) K^{\mathrm{T}}(t)
$$

и через $S^{1 / 2}$ обозначим квадратный корень из $S$. При сделанных обозначениях

$$
P(s+1)=A(s) S(s) A^{\mathrm{T}}(s)+R_{1}(s), \quad s=1,2, \ldots .
$$

Используя свойства 2), 3) следа матрицы и (8), (10), находим

$$
\max _{-(s) \in H_{s}, s=0, t} \mathbf{E}\|\hat{x}(t+1)-x(t+1)\|^{2}=
$$$$
=\max _{\mathbf{A}(s) \in H_{s}, s=0, t} \operatorname{tr}\left(A(t) S(t) A^{\mathrm{T}}(t)+R_{1}(t)\right)=
$$$$
=\max _{A(s) \in H_{s}, s=0, t-1} \max _{A(t) \in H_{t}} \operatorname{tr}\left(S^{1 / 2}(t) A^{\mathrm{T}}(t) A(t) S^{1 / 2}(t)+R_{1}(t)\right)=
$$$$
=\max _{A(s) \in H_{s}, s=0, t-1} \frac{\operatorname{tr}}{\left(S^{1 / 2}(t) A_{0}^{\mathrm{T}}(t) A_{0}(t) S^{1 / 2}(t)+R_{1}(t)\right)=}
$$$$
=\max _{A(s) \in H_{s}, s=0, t-1} \operatorname{tr}\left(A_{0}(t) S(t) A_{0}(t)+R_{1}(t)\right)=
$$$$
=\max _{\boldsymbol{A}(s) \in H_{s}, s=0, t-1} \frac{\operatorname{tr}}{s}\left\{A _ { 0 } ( t ) \left[( I - K ( t ) C ( t ) ) \left(A(t-1) S(t-1) A^{\mathrm{T}}(t-1)+\right.\right.\right.
$$$$
\left.\left.\left.+R_{1}(t-1)\right)(I-K(t) C(t))^{\mathrm{T}}+K(t) R_{2}(t) K^{\mathrm{T}}(t)\right] A_{0}^{\mathrm{T}}(t)+R_{1}(t)\right\} .
$$

Из свойств 4) и 5) матриц следует, что $A_{0}(t)$ максимизирует и все собственные значения матришы $A(t) S(t) A^{\mathrm{T}}(t)$. С учетом представления (11) и свойства 3 ) матриц нетрудно убедиться, что среднеквадратическая ошибка оценки $\hat{x}(t+1)$ монотонно увеличивается при возрастании собственных значений матрицы $A(t-1) S(t-1) A^{\mathrm{T}}(t-1)$. Но, аналогично отмеченному выше, все собственные числа матрицы $A(t-1) S(t-1) \times$ $\times A^{\mathrm{T}}\left((t-1)\right.$ будут максимальными при матрице $A_{0}(t-1)$. Повторяя это рассуждение для $t-2, t-3, \ldots, 0$, получим, что среднеквадрагическая ошибка оценки $\hat{x}(t+1)$ будет максимальной при $A(s)=$ $=A_{0}(s), s=0,1, \ldots, t$. Следовательно, 


$$
\max _{A(s) \in H_{s}, s=0, t} \mathbf{E}\|\hat{x}(t+1)-x(t+1)\|^{2}=\operatorname{tr} P(t+1),
$$

где $P(t)$ определяется следующим рекуррентным уравнением

$$
\begin{gathered}
P(t+1)=A_{0}(t)\left[(I-K(t) C(t)) P(t)(I-K(t) C(t))^{\mathrm{T}}+\right. \\
\left.+K(t) R_{2}(t) K^{\mathrm{T}}(t)\right] A_{0}^{\mathrm{T}}(t)+R_{1}(t) \\
P(0)=R_{0}
\end{gathered}
$$

Таким образом, проблема минимизации максимальной среднеквадратической ошибки оценивания сводится к такому выбору матриц $K(s)$, $s=0,1, \ldots, t$, чтобы минимизировать $\operatorname{tr} P(t+1)$, где $P(t+1)$ определяется уравнением (12). В силу независимости последовательности $\left\{A_{0}(s)\right\}$, которая максимизирует среднеквадратическую ошибку оценивания, от последовательности $\{K(s)\}$ можно использовать результаты, полученные при точно заданных матрицах $A(s)=A_{0}(s), s=0,1, \ldots$. Из $\left[{ }^{5}\right]$ (глава 7$)$ следует, что оптимальная последовательность $\{K(s)\}$ имеет вид

$$
K(s)=P(s) C^{\mathrm{T}}(s)\left(C(s) P(s) C^{\mathrm{T}}(s)+R_{2}(s)\right)^{-1}, \quad s=0,1, \ldots,
$$

где $P(s)$ определяется уравнением (7). Теорема доказана.

П р и м е ры. 1) Пусть известно, что матрицы $A(s), s=0,1, \ldots$, в системе (1) диагональные и их диагональные элементы принадлежат заданным промежуткам. Из доказанной теоремы следует, что минимаксную оценку состояний такой системы получим, если в уравнениях (5) и (6) элементы диагональных матриц $A_{0}(s), s=0,1, \ldots$, выбрать из заданных промежутков с максимальными абсолютными значениями.

2) Пусть матрицы $A(s)$ имеют вид $A(s)=c_{s} \bar{A}(s)$, где матрицы $\bar{A}(s), s=0,1, \ldots$, заданы и информация о скалярной последовательности $\left\{c_{s}\right\}$ ограничивается заданием таких последовательностей $\left\{a_{s}\right\}$, $\left\{b_{s}\right\}$, что $a_{s} \leqslant c_{s} \leqslant b_{s}, s=0,1, \ldots$. Тогда оптимальные $c_{s}{ }^{0}, s=$ $=0,1, \ldots$, определяются как $c_{s}{ }^{0}=a_{s}$, если $\left|a_{s}\right| \geqslant\left|b_{s}\right|$ и $c_{s}{ }^{0}=b_{s}$, если $\left|a_{s}\right|<\left|b_{s}\right|$.

2. Рассмотрим линейный стохастический процесс с непрерывным временем, описываемый уравнениями

$$
\begin{aligned}
& \mathrm{d} x(t)=A(t) x(t) \mathrm{d} t+\mathrm{d} e(t), \\
& \mathrm{d} y(t)=C(t) x(t) \mathrm{d} t+\mathrm{d} v(t), \quad t \in T,
\end{aligned}
$$

где $T=\left[t_{0}, t_{1}\right], x, y$ - векторные функции размерности $n$ и $m$ соответственно, $A, C$ - матричные функции с непрерывными элементами порядка $n \times n$ и $m \times n$. Предположим, что $e$ и $v$ являются стохастическими воздействиями с некоррелированными приращениями, ковариации которых равны $R_{1}(t) \mathrm{d} t$ и $R_{2}(t) \mathrm{d} t$, причем $R_{2}(t), \forall t \in T$, положительно определена и элементы матриц $R_{1}, R_{2}-$ непрерывные функции. Допустим, что начальное состояние $x\left(t_{0}\right)$ системы (13) - случайный вектор с ма. тсматическим ожиданием $x_{0}$, с ковариационной матрицей $R_{0}$ и что процессы $e$ и $v$ взаимно и с $x\left(t_{0}\right)$ не коррелированы.

Пусть функция $C(t)$ в (14) известна, а информация о функции $A(t)$ ограничивается заданием условия $A \in H$, где $H-$ известное множество непрерывных матричных функций, определенное через заданные $n \times n$ матрицы $A_{0}(s), s \in T$, как

$$
H=\left\{A(s): z^{\mathrm{T}}\left(A(s)-A_{0}(s)\right) \quad z \leqslant 0, \forall z \in R^{n}, \forall s \in T\right\} .
$$


По наблюдению за функцией $y(s), s \in\left[t_{0}, t\right]$, нужно оценить вектор состояний $x(t)$. Далее будем предполагать, что допустимые оценки имеют вид

$$
\mathrm{d} \hat{x}(t)=A(t) \hat{x}(t) \mathrm{d} t+K(t)(\mathrm{d} y(t)-C(t) \hat{x}(t) \mathrm{d} t), \quad \hat{x}\left(t_{0}\right)=x_{0},
$$

где $A(s) \in H$ и $K(s)$ - матричная функция порядка $n \times m$ с непрерывными элементами.

Оценку вида (16) будем называть минимаксной, если функции $A(s)$, $K(s)$ определяются из условия

$$
\min _{\boldsymbol{K}(s)} \max _{\boldsymbol{A}(s) \in H} \mathbf{E}\left[(\hat{x}(t)-x(t))^{\mathrm{T}}(\hat{x}(t)-x(t))\right] .
$$

\section{Справедлива}

Т ео рем а 2. Линейная минимаксная оценка состояний системь (13) относительно множества (15) определяется стохастическим дифференциальным уравнением

$$
\begin{aligned}
\mathrm{d} \hat{x}(t) & =A_{0}(t) \hat{x}(t) \mathrm{d} t+P(t) C^{\mathrm{T}}(t) R_{2}^{-1}(t)(\mathrm{d} y(t)-C(t) \hat{x}(t) \mathrm{d} t), \\
\hat{x}\left(t_{0}\right) & =x_{0},
\end{aligned}
$$

где $P(t)$ удовлетворяет уравнению

$$
\begin{aligned}
\frac{\mathrm{d} P(t)}{\mathrm{d} t} & =A_{0}(t) P(t)+P(t) A_{0}^{\mathrm{T}}(t)+R_{1}(t)-P(t) C^{\mathrm{T}}(t) R_{2}^{-1}(t) C(t) P(t), \\
P\left(t_{0}\right) & =R_{0},
\end{aligned}
$$

при этом

$$
\operatorname{tr} P(t)=\min _{K} \max _{A \in H} \mathbf{E}\|\hat{x}(t)-x(t)\|^{2}
$$

Д о к а 3 а те льс тв о. Обозначим $\tilde{x}=\hat{x}-x$. Используя (13) и (16), получим $\tilde{x}$ уравнение

$$
\begin{aligned}
\mathrm{d} \widetilde{x}(t) & =(A(t)-K(t) C(t) \tilde{x}(t) \mathrm{d} t+K(t) \mathrm{d} v(t)-\mathrm{d} e(t), \\
\tilde{x}\left(t_{0}\right) & =0 .
\end{aligned}
$$

Согласно теореме 6.1 из $\left[{ }^{5}\right]$ (глава 3 ), ковариационная матрица $P(t)$ ошибки $\tilde{x}(t)$ определяется следующим дифференциальным уравнением

$$
\begin{aligned}
\frac{\mathrm{d} P(t)}{\mathrm{d} t}=(A(t)-K(t) C(t)) P(t)+P(t)(A(t)-K(t) C(t))^{\mathrm{T}}+ \\
\quad+K(t) R_{2}(t) K^{\mathrm{T}}(t)+R_{1}(t), \\
P\left(t_{0}\right)=R_{0} .
\end{aligned}
$$

Ясно, что

$$
\mathbf{E}\|\hat{x}(t)-x(t)\|^{2}=\mathbf{E}\left(\tilde{x}^{\mathrm{T}}(t) \tilde{x}(t)\right)=\operatorname{tr} P(t) .
$$

Учитывая $(20)$ и линейность следа матрицы, находим

$$
\begin{aligned}
& \frac{\mathrm{d}(\operatorname{tr} P(t))}{\mathrm{d} t}=\operatorname{tr}\left[2(A(t)-K(t) C(t)) P(t)+K(t) R_{2}(t) K^{\mathrm{T}}(t)+R_{1}(t)\right], \\
& \quad \operatorname{tr} P\left(t_{0}\right)=\operatorname{tr} R_{0} .
\end{aligned}
$$


Исследуем максимум функции на правой стороне равенства (21). Используя свойства 2), 3) следа матрицы, имеем

$$
\begin{aligned}
& \max _{A(s) \in H} \operatorname{tr}\left[2(A(s)-K(s) C(s)) P(s)+K(s) R_{2}(s) K^{\mathrm{T}}(s)+R_{1}(s)\right]= \\
= & \max _{A(s) \in H} \operatorname{tr}\left(2 P^{1 / 2}(s) A(s) P^{1 / 2}(s)-2 K(s) C(s) P(s)+\right. \\
+ & \left.K(s) R_{2}(s) K^{\mathrm{T}}(s)+R_{1}(s)\right)= \\
= & \operatorname{tr}\left[2\left(A_{0}(s)-K(s) C(s)\right) P(s)+K(s) R_{2}(s) K^{\mathrm{T}}(s)+R_{1}(s)\right], s \in\left[t_{0}, t\right] .
\end{aligned}
$$

Из теоремы (в) в $\left[{ }^{6}\right]$ (глава I, § 2, п. 8) выводится, что

$$
\left.\operatorname{tr} P(s)\right|_{A \in H} \leqslant\left.\operatorname{tr} P(s)\right|_{A=A_{0}}, \quad s \in\left[t_{0}, t\right] .
$$

Следовательно,

$$
\max _{A \in H} \mathbf{E}\|\hat{x}(t)-x(t)\|^{2}=\operatorname{tr} P(t)
$$

где матрица $P(t)$ определяется дифференциальным уравнением

$$
\begin{aligned}
& \frac{\mathrm{d} P(t)}{\mathrm{d} t}=\left(A_{0}(t)-K(t) C(t)\right) P(t)+P(t)\left(A_{0}(t)-K(t) C(t)\right)^{\mathrm{T}}+ \\
& \quad+K(t) R_{2}(t) K^{\mathrm{T}}(t)+R_{1}(t), \\
& P\left(t_{0}\right)=R_{0} .
\end{aligned}
$$

Поскольку выбор оптимальной функции $A_{0}$ независимый от функции $K$, то при минимизации по $K$ максимальной среднеквадратической ошибки (22) можем использовать результаты, полученные при точно заданной функции $A=A_{0}$. Из $\left[{ }^{5}\right]$ (глава 7) следует, что минимизирующей функцией является $K(t)=P(t) C^{\mathrm{T}}(t) R_{2}{ }^{-1}(t)$, где $P(t)$ определяется уравнением (18). Следовательно, минимаксная оценка $\hat{x}(t)$ удовлетворяет уравнению (17). Теорема доказана.

В частном случае, когда в системе (13) матрицы $A(t)$ диагональные, $A(t)=\left(a_{i}(t), i=\overline{1, n}\right), \forall t \in T$, и информация о скалярных функциях $a_{i}(t)$ ограничивается заданием таких непрерывных функций $a_{i}{ }^{0}(t)$, что $a_{i}(t) \leqslant a_{i}{ }^{0}(t), \forall t \in T, i=\overline{1, n}$, то минимаксная оценка состояний этой системы удовлетворяет уравнению (17), где $A_{0}(t)=\left(a_{i}{ }^{0}(t), i=\overline{1, n}\right)$, $\forall t \in T$.

\section{ЛИТЕРАТУ Р А}

1. Наконечный $A . \Gamma$. В кн.: Теория вероятностей и математической статистики. Қинев, $18,115-120(1978)$.

2. Krener, A. J. IEEE Trans. Autom. Control, 25, № 2, 291-292 (1980).

3. Poor, V., Looze, D. P. IEEE Trans. Autom. Control, 26, № 4, 902-906 (1981).

4. Vaatmann, V. ENSV TA Toim. Füüs. Matem. 28, № 4, 365-367 (1979)

5. Asström, $K J$. Introduction to Stochastic Control Theory. New York, Academic Press, 1970.

6. Камке Э. Справочник по обыкновенным дифференциальным уравнениям. М., «Наука», 1965.

Институт кибернетики Академии наук Эстонской ССР

Поступнла в редакцию 11/IV 1983 


\section{LINEAARSE STOHHASTILISE SUSTEEMI OLEKUFUNKTSIOONI MINIMAKSHINDAMISEST}

Artiklis on uuritud stohhastilise süsteemi olekufunktsiooni lineaarset minimakshindamist olukorras, kus selle süsteemi üleminekumaatriksid ei ole täpsemalt teada. Vaadeldud juhtudel avaldub minimakshinnang Kalman-Bucy võrrandi tüüpi rekurrentsete võrranditena.

\section{VAATMANN}

\section{ON THE MINIMAX STATE ESTIMATION FOR LINEAR STOCHASTICAL SYSTEMS}

The minimax state estimation for discrete time (1) and continuous time (13) processes when the transitional matrices are not exactly known, is considered. In the discrete time case it is assumed that the matrix sequence $A(s)$ is contained in the given set (3) and the recursive equation (5) for the minimax estimate relative to the (4) is obtained.

In the continuous time case it is assumed that the matrix function $A(s)$ is constrai$\mathrm{n}$ d with the set (15). The minimax state estimate satisfies the stochastic differential cquation (17). 